Z. klin. Chem. u. klin. Biochem.

8. Jg., S. $618-620$, November 1970

\title{
Zur hormonalen Regulation des Zn-Stoffwechsels
}

\author{
Von F. Dorn und Th. GÜNTHeR \\ Aus dem Plyysiologiscb-chemischen Institut der Freien Universität Berlin
}

(Eingegangen am 30. Juli 1970)

Von allen untersuchten hormonalen Zuständen war nach Adrenalektomie der Zn-Gehalt in Leber und Niere am stärksten erhöht. Die Wirkung der Adrenalektomie auf den Zn-Gehalt der Leber konnte durch Cortison, nicht aber durch Aldosteron ausgeglichen werden. Die Organe, deren $\mathrm{Zn}$-Gehalt nach Adrenalektomie erhöht ist, nehmen mehr ${ }^{65} \mathrm{Zn}$ auf, bei den anderen ist der ${ }^{65} \mathrm{Zn}$-turnover verlangsamt.

\section{The bormonal regulation of $Z n$ metabolism}

The concentration of $\mathrm{Zn}$ in the liver and kidneys was determined in various hormonal states; the greatest increase was caused by adrenalectomy. The effect of adrenalectomy on the $\mathrm{Zn}$ concentration of the liver was reversed by cortisone, but not by aldosterone. Organs that show an increased $\mathrm{Zn}$ concentration after adrenalectomy take up more ${ }^{65} \mathrm{Zn}$ under normal conditions than do the other organs, where the rate of turnover of ${ }^{65} \mathrm{Zn}$ is lower.

Zn gehört zu den lebensnotwendigen Elementen. Es konnte als Bestandteil verschiedener Enzyme (NADabhängige : Dehydrogenasen, Phosphatase, Carboanhydratase, Carboxypeptidase) nachgewiesen werden (1) und soll die Biosynthese von Proteinen und Nucleinsäuren stimulieren (2).

Bei $\mathrm{Zn}$-armer Ernährung treten bei erwachsenen Tieren mannigfaltige Mangelerscheinungen (1) und bei Foeten Mißbildungen auf $(3,4)$. Bei verschiedenen Krankheiten ändert sich die Zn-Konzentration im Serum (5) u. a. bei endokrinen Störungen. So wurde eine Abnahme der Serum-Zn-Konzentration bei Hypophyseninsuffizienz, M. Addison und. Myxödem, eine $\mathrm{Zu}$ nahme bei Hyperthyreose oder nach Injektion von Adrenalin, Thyroxin und thyreotropem Hormon (5) gefunden. Eine (direkte oder indirekte) hormonale Regulation des $\mathrm{Zn}$-Stoffwechsels oder eine Beteiligung des $\mathrm{Zn}$ am Zustandekommen dieser krankhaften Störungen würden entsprechende Änderungen des intrazellulären $\mathrm{Zn}$-Gehalts erwarten lassen, zumal sich etwa $99 \%$ des $\mathrm{Zn}$-Gehalts eines Organs in den Zellen befinden. Wir haben deshalb den Einfluß von Hormonen auf den $\mathrm{Zn}$-Gehalt in verschiedenen Organen untersucht.

\section{Methodik}

Die Vorbehandlung der männlichen Albinoratten und der in Äthernarkose entnommenen Organe wurde früher ausführlich beschrieben (6). Zur Bestimmung des $\mathrm{Zn}$-Gehalts wurde Serum im Verhältnis 1:4 mit bidest. Wasser, verdünnt. Von den gefriergetrockneten Organen wurden etwa $50 \mathrm{mg}$ mit $0,5 \mathrm{ml}$ konz. $\mathrm{HNO}_{3} 1 \mathrm{Std}$. im siedenden Wasserbad erhitzt und mit $5 \mathrm{~m} l$ bidest. Wasser versetzt. Die $\mathrm{Zn}$-Bestimmung wurde im Atomabsorptionsspektrophotometer (M4QIII-FA2, Fa. Zeiss) durchgeführt.

Zur Bestimmung des $\mathrm{Zn}$-Fluxes wurden $10 \mu \mathrm{C}{ }^{65} \mathrm{Zn} / 100 \mathrm{~g}$ Ratte i. v. injiziert ${ }^{65} \mathrm{Zn}, 100-2500 \mathrm{mC} / \mathrm{g} \mathrm{Zn}$, Radiochemical Centre Amersham).

$\mathrm{Zu}$ verschiedenen Zeiten wurden den Tieren in Äthernarkose Blut und Organe entnommen. In $0,1-1 \mathrm{~m} l$ Serumproben bzw. $20-50 \mathrm{mg}$ Organtrockensubstanz nach Veraschung mit $\mathrm{HNO}_{3}$ wurde die Radioaktivität des ${ }^{65} \mathrm{Zn}$ im Bohrlochktistall (Fa. Frieseke u. Hoepfner) gemessen, Meßvolumen jeweils $1 \mathrm{ml}$. Bei der graphischen Darstellung der ${ }^{65} \mathrm{Zn}$-Aufnahme in die Gewebe wurde jeweils die Aktivität des extrazellulären $\mathrm{Zn}$ abgezogen. Hierfür wurde das Verteilungsvolumen des extrazellulären ${ }^{65} \mathrm{Zn}$ in den Organen mit 10\%, im Muskel mit seiner geringeren extrazellulären Flüssigkeit (EZF) mit $5 \%$ und im Knochen mit $0 \%$ angenommen. Eine Rechnung zeigt, daß der Fehler durch die Ungenauigkeit dieser Größe unerheblich ist.

\section{Ergebnisse und Diskussion}

Tabelle 1 zeigt, daß Hypophysektomie, STH-Behandlung und die Sexualhormone den $\mathrm{Zn}$-Gehalt von Leber und Muskulatur nicht signifikant beeinflussen.

Tab. 1

Zn-Gehalt ( $\bar{x} \pm$ mittlerer Fehler des Mittelwertes) in Lebern und Muskeln bei verschiedenen hormonalen Zuständen. [Die Tiere sind identisch mit (6), dort auch weitere Angaben]

\begin{tabular}{|c|c|c|}
\hline & $\mathrm{Zn}[\underset{\text { Leber }}{\mathrm{mMol} / \mathrm{kg}}$ & $\begin{array}{c}\text { Trockensubstanz] } \\
\text { Muskel }\end{array}$ \\
\hline Normale Tiere & $1,57 \pm 0,014$ & $0,812 \pm 0,023$ \\
\hline Hypophysektomierte Tiere & $1,61 \pm 0,040$ & $0,840 \pm 0,042$ \\
\hline Normale Tiere + STH & $1,64 \pm 0,072$ & $0,845 \pm 0,032$ \\
\hline Adrenalektomierte Tiere & $2,70 \pm 0,207$ & $0,843 \pm 0,077$ \\
\hline Adrenalektomierte $\mathrm{T}$. + Aldoster. & $2,47 \pm 0,148$ & $0,864 \pm 0,015$ \\
\hline Adrenalektomierte $\mathrm{T}$. + Cortison & $1,48 \pm 0,054$ & $1,03 \pm 0,033$ \\
\hline Normale T. + Methylthiouracil & $1,64 \pm 0,029$ & $1,42 \pm 0,065$ \\
\hline Normale $\mathrm{T} .+$ Thyroxin & $1,33 \pm 0,064$ & $0,901 \pm 0,030$ \\
\hline Parathyreoidektomierte Tiere & $1,85 \pm 0,094$ & $0,841 \pm 0,042$ \\
\hline Kastrierte Tiere & $1,59 \pm 0,039$ & $0,780 \pm 0,019$ \\
\hline Normale Tiere + Testosteron & $1,58 \pm 0,065$ & $0,778 \pm 0,028$ \\
\hline Normale Tiere + Ostradiol & $1,64 \pm 0,059$ & $0,739 \pm 0,021$ \\
\hline Normale Tiere + Progesteron & $1,66 \pm 0,028$ & $0,643 \pm 0,017$ \\
\hline
\end{tabular}

Nach Adrenalektomie war der Zn-Gehalt in der Leber um etwa $70 \%$ höher als bei Normaltieren. Der $\mathrm{Zn}$ Gehalt der Muskulatur war unverändert. Aldosteronbehandlung hatte keinen Einfluß auf die Verteilung des $\mathrm{Zn}$. Cortisonbehandlung dagegen verbinderte das Ansteigen des $\mathrm{Zn}$-Gehalts in der Leber, bewirkte aber im Muskel - möglicherweise infolge der hohen Dosierung - eine geringe Erhöhung des Zn. Nach Entfernung der Epithelkörperchen war der $\mathrm{Zn}$-Gehalt in der Leber etwas erhöht. Methylthiouracil-Gabe 
führte nur im Muskel zur Vermehrung des $\mathrm{Zn}-\mathrm{Be}$ standes, Thyroxin bewirkte dagegen eine geringe Senkung des $\mathrm{Zn}$-Gehalts in der Leber.

Diese Veränderungen sind mit den von Wolfr (5) beschriebenen Hormon-abhängigen Veränderungen der Serum-Zn-Konzentration korrelierbar. Danach könnte eine Zunahme des Serum-Zn bei Hyperthyreose durch Austreten von $\mathrm{Zn}$ aus der Leberzelle und die Abnahme beim Myxödem auf einer Aufnahme von $\mathrm{Zn}$ in die Muskelzelle beruhen.

Die größten Änderungen der Zn-Verteilung erfolgten nach Adrenalektomie. Ihre Normalisierung durch Cortison läßt den Schluß zu, daß die normale $\mathrm{Zn}$ Verteilung durch Glucocorticoide reguliert wird. Wir haben deshalb die Wirkung der Adrenalektomie auf den Zn-Stoffwechsel näher untersucht (Tab. 2).

Die Zunahme des Zn-Gehalts in der Leber ist bereits 2 Tage nach der Adrenalektomie nachzuweisen. Eine signifikante Zunahme fanden wir außerdem noch in der Niere. In den anderen untersuchten Organen blieb der Zn-Gehalt gleich. Die Beschränkung des Effektes auf Leber und Nieren läßt sich mit einer stärkeren Beeinflussung dieser Organe durch Glucocorticoide erklären, denn sie nehmen von zugegebenem ${ }^{14} \mathrm{C}$-Cortisol auch am meisten auf (7).

Im Serum und im Knochen nahm der Zn-Gehalt etwa im gleichen Verhältnis ab. Das weist darauf hin, daß $\mathrm{Zn}$ im Knochen an Apatit adsorbiert ist und mit dem Zn der EZF im Gleichgewicht steht.

Eine bilanzmäßige Betrachtung zeigt, daß die $\mathrm{Zn}$ Menge, die nach Adrenalektomie den Knochen verlassen hat, etwa der $\mathrm{Zn}$-Zunahme in Leber und Nieren entspricht. Nach Adrenalektomie gelangt offenbar zunnächst $\mathrm{Zn}$ aus der EZF in Leber- und Nierenzellen. Dadurch vermindert sich die extrazelluläre $\mathrm{Zn}-\mathrm{Kon}$ zentration, und $\mathrm{Zn}$ wird im Knochen desorbiert.

Zur Erklärung des höheren Zn-Gehalts in Leber und Nieren nach Adrenalektomie bestimmten wir die Geschwindigkeit, mit der ${ }^{65} \mathrm{Zn}$ in verschiedene Organe aufgenommen wird. 5 Min. nach i. v.-Injektion von ${ }^{65} \mathrm{Zn}$; wenn die Verteilung in der EZF der Ratte abgeschlossen ist, ist die $\mathrm{Zn}$-Aktivität im Serum adrenalektomierter Ratten um etwa $20 \%$ höher als bei den Kontrollen (Abb. 1). Dies läßt sich damit erklären, daß die EZF nach Adrenalektomie verringert ist. Die ${ }^{65} \mathrm{Zn}$-Aktivität im Serum nimmt danach bis zur untersuchten Zeit (8 Stdn. nach Injektion) in einer zweigliedrigen Exponentialfunktion $\mathrm{ab}$. $\mathrm{Da}$ während dieser Zeit die durch Nieren und Darm ausgeschiedene $\mathrm{Zn}$ Menge gering ist (8), kann man die beiden Glieder der Exponentialfunktion auf eine schnelle und eine langsame $\mathrm{Zn}$-Permeation in den intrazellulären Raum zurückführen. Abbildung 2 zeigt, daß für die schnelle ${ }^{65} \mathrm{Zn}$-Aufnahme hauptsächlich Leber und Nieren, für die langsame Aufnahme die übrigen untersuchten Organe, Muskel, Herz, Hoden und Knochen in Frage kommen. Der zeitliche Verlauf der ${ }^{65} \mathrm{Zn}$-Aufnahme und Abgabe stimmt bei den verschiedenen Organen der
Kontrolltiere mit früheren Angaben über die ${ }^{65} \mathrm{Zn}-$ Aufnahme überein (9).

Berechnet man die relative spezifische Aktivität (Imp./ Min. pro g Organ/ $\mu \mathrm{Mol} \mathrm{Zn}$ pro g Organ/Imp./Min. pro $\mathrm{ml}$ Serum/ $\mu \mathrm{Mol} \mathrm{Zn}$ pro $\mathrm{ml}$ Serum), so ergibt sich, $\mathrm{daB}$ der $\mathrm{Zn}$-Austausch in Leber und Nieren schnell und vollständig erfolgt. Erst nach $8 \mathrm{Stdn}$. ist das $\mathrm{Zn}$ im Herzmuskel zu 100\%, im Skelettmuskel zu 30\%, im Hoden zu 25\% und im Knochen zu 5\% ausgetauscht. $\mathrm{Zu}$ dieser Zeit ist die relative spezifische $\mathrm{Ak}$ tivität in Leber und Nieren $>1$, d. h. der Rücktausch

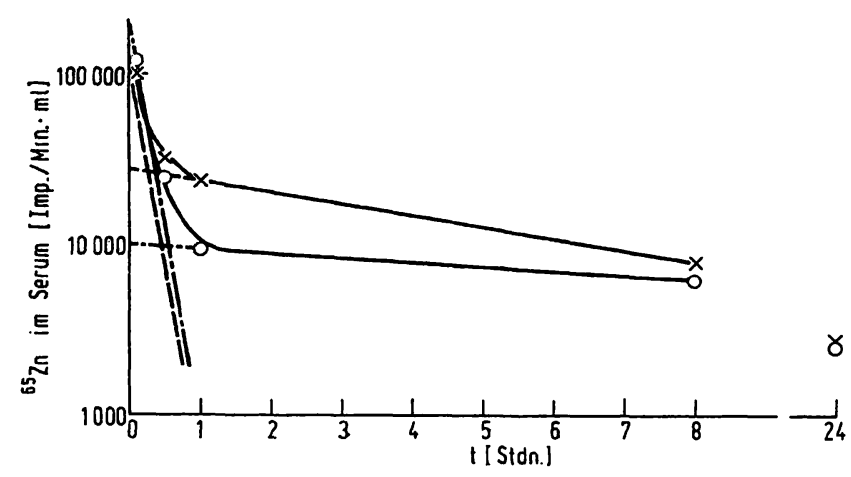

Abb. 1

oszn-Aktivität im Serum normaler $(x)$ und adrenalektomierter (o) Ratten (3 Tage nach Adrenalektomie) zu verschiedenen Zeiten nach i. v. Injektion von $10 \mu \mathrm{C}{ }^{\circ} \mathrm{Z} \mathrm{Z} / 100 \mathrm{~g}$ Ratte (Mittelwerte von je 4 Tieren)
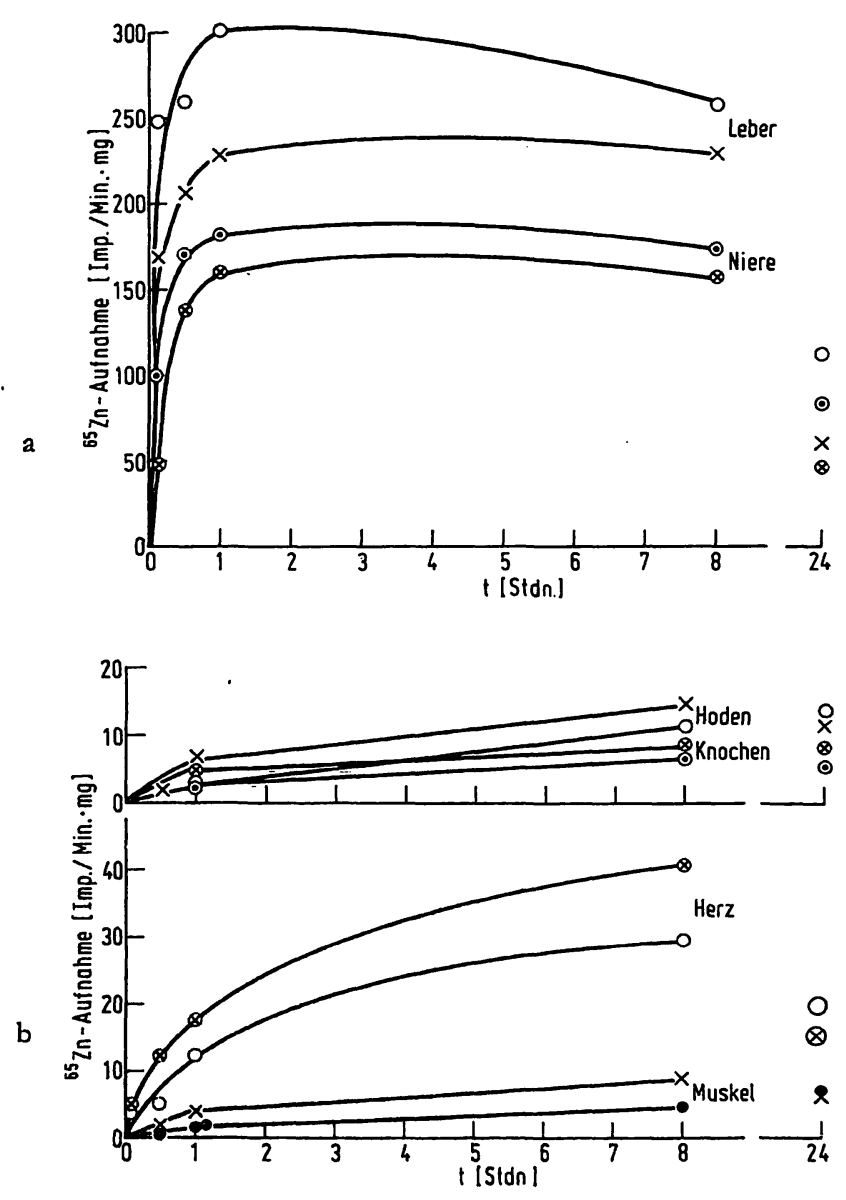

Abb. 2a und b

Aufnahme von ${ }^{\circ} \mathrm{Zn}$ in verschiedene Organe, bezogen auf Frischgewicht, Kontrollen: $x, \otimes$, adrenalektomierte Tiere: $\bullet, 0, \odot$ (Mittelwerte von je 4 Tieren) 
Tab. 2

$\mathrm{Zn}-\mathrm{Gehalt}$ in Serum und verschiedenen gefriergetrockneten Organen von normalen, mit Cycloheximid behandelten [3 Tage lang, täglich $0,2 \mathrm{mg} /$ 100 intraperion

Adrenalektomierte Ratten

\begin{tabular}{|c|c|c|c|c|c|}
\hline & \multirow{2}{*}{$\begin{array}{c}\text { Normale Ratten, } \\
\text { Cycloheximid }\end{array}$} & \multirow[b]{2}{*}{$\begin{array}{c}\text { Normale Ratten, } \\
\text { unbehandelt }\end{array}$} & \multicolumn{3}{|c|}{ Adrenalektomierte Ratten } \\
\hline & & & 2 Tage nach Op. & 5 Tage nach Op. & 10 Tage nach Op. \\
\hline $\begin{array}{l}\text { Serum }(\mu \mathrm{Mol} / \mathrm{l}) \\
\text { Leber } \mathrm{mMol} / \mathrm{kg} \text { Trockensubstanz } \\
\text { Niere mMol/kg Trockensubstanz } \\
\text { Herz mMol/kg Trockensubstanz } \\
\text { Muskel mMol/kg Trockensubstanz } \\
\text { Hoden mMol } / \mathrm{kg} \text { Trockensubstanz } \\
\text { Knochen mMol } / \mathrm{kg} \text { Trockensubstanz }\end{array}$ & $\begin{array}{r}21,8 \pm 0,60 \\
2,01 \pm 0,08 \\
1,40 \pm 0,06 \\
1,17 \pm 0,02 \\
0,680 \pm 0,02 \\
2,70 \pm 0,05 \\
2,22 \pm 0,05\end{array}$ & $\begin{array}{r}33,4 \pm 0,77 \\
1,37 \pm 0,02 \\
1,25 \pm 0,01 \\
0,963 \pm 0,004 \\
0,564 \pm 0,004 \\
2,75 \pm 0,013 \\
2,79 \pm 0,067\end{array}$ & $\begin{array}{r}25,7 \pm 1,09 \\
2,26 \pm 0,04 \\
1,52 \pm 0,04 \\
1,01 \pm 0,01 \\
0,617 \pm 0,01 \\
2,74 \pm 0,01 \\
2,52 \pm 0,08\end{array}$ & $\begin{array}{l}31,44 \pm 0,59 \\
1,995 \pm 0,02 \\
1,87 \pm 0,06 \\
0,987 \pm 0,01 \\
0,572 \pm 0,004 \\
2,76 \pm 0,04\end{array}$ & $\begin{array}{r}27,95 \pm 0,37 \\
1,82 \pm 0,04 \\
1,93 \pm 0,03 \\
1,01 \pm 0,01 \\
0,574 \pm 0,01 \\
2,68 \pm 0,02 \\
2,43 \pm 0,11\end{array}$ \\
\hline
\end{tabular}

Tab. $2 a$

Wassergehalt (\%) der untersuchten Organe (vgl. Tab. 2)

\begin{tabular}{|c|c|c|c|c|c|}
\hline & \multirow[b]{2}{*}{$\begin{array}{l}\text { Normale Ratten, } \\
\text { Cycloheximid }\end{array}$} & \multirow[b]{2}{*}{$\begin{array}{l}\text { Normale Ratten, } \\
\text { unbehandelt }\end{array}$} & \multicolumn{3}{|c|}{ Adrenalektomierte Ratten } \\
\hline & & & 2 Tage nach Op. & 5 Tage nach Op. & 10 Tage nach $O p$. \\
\hline $\begin{array}{l}\text { Leber } \\
\text { Niere } \\
\text { Herz } \\
\text { Muskel } \\
\text { Hoden } \\
\text { Knochen }\end{array}$ & $\begin{array}{l}71,30 \pm 0,34 \\
75,70 \pm 0,69 \\
75,18 \pm 0,30 \\
75,12 \pm 0,26 \\
85,96 \pm 0,46 \\
33,30 \pm 0,60\end{array}$ & $\begin{array}{l}68,22 \pm 0,19 \\
74,44 \pm 0,12 \\
76,62 \pm 0,21 \\
74,42 \pm 0,08 \\
85,98 \pm 0,13 \\
25,9 \pm 0,71\end{array}$ & $\begin{array}{l}71,7 \pm 0,25 \\
75,23 \pm 0,43 \\
76,68 \pm 0,21 \\
74,98 \pm 0,22 \\
85,82 \pm 0,10 \\
27,8 \pm 0,8\end{array}$ & $\begin{array}{l}70,9 \pm 0,33 \\
74,90 \pm 0,10 \\
76,12 \pm 0,04 \\
74,74 \pm 0,22 \\
85,72 \pm 0,11 \\
\end{array}$ & $\begin{array}{l}71,84 \pm 0,20 \\
75,85 \pm 0,23 \\
76,34 \pm 0,10 \\
75,4 \pm 0,07 \\
.86,10 \pm 0,16 \\
28,9 \pm 0,7\end{array}$ \\
\hline
\end{tabular}

des ${ }^{65} \mathrm{Zn}$ erfolgt in diesen Organen langsamer als die ${ }^{65} \mathrm{Zn}$-Elimination aus dem Serum.

Aus Abbildung 1 geht weiter hervor, daß bei adrenalektomierten Ratten die ${ }^{65} \mathrm{Zn}$-Aktivität im Serum zunächst schneller und stärker abnimmt als bei den Kontrollen, d.h. bei den adrenalektomierten Tieren wird in der ersten Phase $\mathrm{Zn}$ mit etwas höherer Geschwindigkeit in einen größeren Verteilungsraum aufgenommen. Eine Analyse der Exponentialfunktionen ergibt für den schnellen Aktivitätsabfall bei den Kontrolltieren eine Halbwertszeit von etwa $10 \mathrm{Min}$. und bei den adrenalektomierten Tieren von etwa 7 Min. Dieser schnelleren Elimination von ${ }^{65} \mathrm{Zn}$ aus dem Serum adrenalektomierter Ratten entspricht eine schnellere ${ }^{65} \mathrm{Zn}$-Aufnahme in Leber und Nieren bei diesen Tieren (Abb. 2). Die höhere ${ }^{65} \mathrm{Zn}$-Aktivität in Leber und Nieren läßt sich auf den höheren Zn-Gehalt dieser Organe nach Adrenalektomie zurückführen.

Das zweite Glied der Exponentialfunktionen der Abbildung 1 ergibt bei den Kontrolltieren eine Halbwertszeit von 4,5 Stdn., bei den adrenalektomierten Tieren von etwa $9 \mathrm{Stdn}$. Während der zweiten Phase nimmt also die ${ }^{65} \mathrm{Zn}$-Aktivität im Serum adrenalektomierter Ratten langsamer ab. Hiermit stimmt überein, $\mathrm{daB}$ bei diesen Tieren ${ }^{65} \mathrm{Zn}$ mit geringerer Geschwindigkeit in Herz, Skelettmuskel, Hoden und Knochen aufgenommen wird.

Vergleicht man die Geschwindigkeiten der Zn-Aufnahme in die verschiedenen Organe, so zeigt sich, daß sie nicht mit dem $\mathrm{Zn}$-Gehalt korreliert sind. Für die bei verschiedenen Organen unterschiedliche Aufnahmegeschwindigkeit scheint daher ein membrangebundener Transportmechanismus verantwortlich zu sein.

Der höhere Zn-Gehalt von Leber und Nieren nach Adrenalektomie kann durch eine Zunahme von intrazellulären Zn-Komplexbildnern oder durch eine Änderung der Geschwindigkeitskonstanten für den $\mathrm{Zn}$ Influx und Zn-Efflux bedingt sein.

Bei den Organen mit gleichbleibendem Zn-Gehalt und nach Adrenalektomie verlangsamtem ${ }^{65} \mathrm{Zn}$-Influx kann man auf einen im gleichen Maße verlangsamten $\mathrm{Zn}$ Efflux schließen.

Ein verminderter Efflux und seine Erhöhung durch Glucocorticoide wurde auch für das nach Adrenalektomie vermehrte $\mathrm{Cu}$ in der Leber angenommen (10). An der Elimination von $\mathrm{Cu}$ aus der Leber ist wahrscheinlich Protein beteiligt, denn nach Hemmung der Proteinsynthese war der Cu-Gehalt in der Leber erhöht (11). Die Synthese dieses Proteins soll ebenfalls durch Nebennierenrindenhormone beeinflußt werden (10). Beim Zn könnten ähnliche Beziehungen bestehen, denn nach Gaben von Cycloheximid, wodurch die Proteinsynthese gehemmt wird, war der Zn-Gehalt in Serum und Knochen (wie nach Adrenalektomie) abgesunken und vor allem in der Leber ebenfalls angestiegen (Tab. 2). Für eine Analogie spricht weiter, daß beide Metalle im Cytoplasma von Leber und Nieren größtenteils an ein Protein vom Molgewicht etwa 10000 gebunden sind (12).

\section{Literatur}

1. VAllee, B. L., in: C.L. Comar and F. Bronner, Mineral Metabol. Bd. 2B, S. 443 Acad. Press New York, London (1962). - 2 Weser, U., S. Seeber und P. WArnecke, Z. Naturforsch. 24b, 866 (1969). - 3. Hurlex, L. S., Fed. Proc. 27, 193 (1968). - 4. Diamond, I. und I. S. Hurlex, J. Nutrit. 100, 325 (1970). 5. Wolfr, H. P., Klin. Wschr. 34, 409 (1956). - 6. GÜNTTHER, Th. und CH. Alter, diese Z. 5, 67 (1967). - 7. BeLlaMY, D., J. G. Phillips, T. C. Jones und R. A. Leonard, Biochem. J.
85, 537 (1962). - 8. Sheline, G. E., I.I. Chatgoff, H. D. Jones und L. M. Montgomerx, J. biol. Chemistry 147, 409 (1943). 9. Sheline, G. E., I. I. Chaigoff, H. D. Jones und L. M. MontGOMERY, J. biol. Chemistry 149, 139 (1943). - 10. GREGORIADIS, G. und T. L. Sourkes, Canad. J. Biochem. 48, 160 (1970). 11. Gregoriadis, G. und T. L. Sourkes, Nature, London 218 , 290 (1968). - 12. Evans, C. W. und W. E. Cornatzer, Fed. Proc. 29, 695 (1970).

Prof. Dr. Th. Günther, 1000 Berlin 33, Arnimallee 22 


\section{Wie lange dauert bei Ihnen eine wahre Glukose-Bestimmung?}

\section{Wir benötigen nur 10 Sekunden ...}
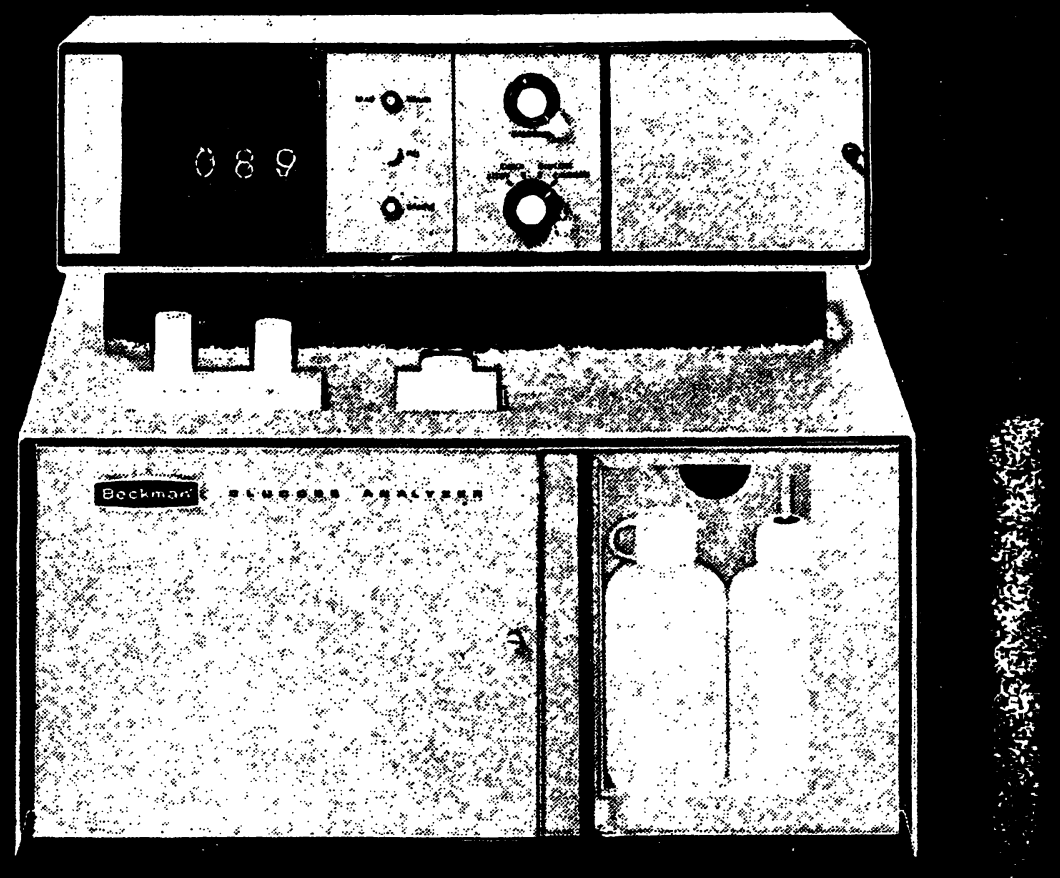

... mit dem neuen Beckman-Glukose-Analysator Modell ERA-2001 durch Anwendung einer völlig neuen Meßtechnik.

Unser Gerät ermittelt die wahre Glukose durch polarographische Messung der Abnahmegeschwindigkeit des Sauerștoffs während der enzymatischen Oxydation.

Weitere überzeugende Argumente:

2. Mikroliter-Proben

$10 \mu \mathrm{l}$ Serum oder Plasma bzw. $50 \mu \mathrm{l}$ Urin

(3) Ohne Enteiweißung oder Probenverdünnung
오 Digitale Anzeige der Meßwerte in $\mathrm{mg} \%$ Glukose

Meßgenauigkeit $\pm 2 \%$ Linearität der Anzeige bis $400 \mathrm{mg} \%$

Außerordentlich günstiger Preis

Bitte besuchen Sie uns auf der ANALYTICA in München vom 29. April bis 2. Mai 1970.

Sie finden uns in Halle 3, Stand-Nr. 3115-19, 3214-20.

\section{Beckman $^{\oplus}$}

BECKMAN INSTRUMENTS GMBH 8 München 45, Frankfurter Ring 115, Tel. 38871 , Telex 05-23823

Technische Büros: Berlin, Tel. 31210 35; Hamburg, Tel. 5195 54; HanTechnische Büros: Berlin, Tel. 31210 35; Hamburg, Tel. 519554 ; Han-
nover, Tel. 66 39 92; Düsseldorf, Tel. 6844 93; Frankfurt, Tel. (06103) 10 03; ntuttgart, Tel. 71 18 37; München, Tel. 885035

Internationale Niederlassungen: Fullerton/USA, Genf, Paris,

Glenrothes/Schottland, Tokio, Kapstadt, Wien, Amsterdam, Stockholm 


\title{
ACTA BIOCHIMICA ET BIOPHYSICA \\ ACADEMIAE SCIENTIARUM HUNGARICAE
}

\author{
(iIm \\ Zeitschrift der Ungarischen Akademie der Wissenschaften'
}

VOLUME 5

NUMBER 2

\section{Contents}

S. Fazekas, M. Nagy, Vilma Székessy-Hermann, E. Wolfram: Viscometric Investigation of Actin G and Actin F

Á. Furka, F. Sebestyén: Studies on Carboxyl Modified Proteins and their Enzymetic Hydrolysates

L. Patrhy, G. DÉNes: Altered Repression Behaviour in a Feedback Insensitive Mutant of Eschericbia coli K12

J. SüDI, M. G. KhAN: Factors Affecting Freesing-Induced Transient Dissociation of Lactate Dehydrogenase Tetramers

Ágnes Déri, Mária Woldenann: Simulation by Analogue Computer of the Reaction Catalysed by Alcohol Dehydrọgenase

Piroska Hüvös, L. Vereczkey, Ö. GaÁl: Effect of Ribonuclease on Pigeon Liver Ribosomal Ribonucleic Acids

Piroska Hüvös, L. Vereczkey, Ö. GaÁL, Mária SzÉkely: RNase Sensitivity of Messenger and Ribosomal RNA in Pigeon Liver Polysomes

MÁriA VAS, L. Boross: Characterization of the Less-reactive SH Groups of D-glyceraldehyde-3-phosphate Dehydrogenase. I. Kinetic Analysis of Mercaptide Formation

MÁrIA VAS, L. Boross: Characterization of the Less-reactive SH Groups of D-glyceraldehyde-3-phosphate Dehydrogenase. II. Effect of Coenzyme, Anions and $\mathrm{pH}$ on the Reactivity

P. Závodszky, P. Elődr: Structural Investigations on Pancreatic $\alpha$-Amylase. II. Determination of the Molecular Weight by Sedimentation and Light Scattering

M. SAJGó: Identification of 1-Dimethylamino-naphthalene-5-sulfonyl Amino Acids by Thin Layer Electrophoresis (Short Communication)

ANNa Gulyás, F. Solymosy: Effect of Diethyl Pyrocarbonate on the Biological Activity of Intact TMV-RNA (Preliminary Communication)

E. LÁbos, Gy. Fazekas: On the Dynamics of Accommodation for the Muscle-response of Anodonta Larva and the Electric Response of Anodonta Nerve

J. Holland, F. Anton: Effect of Heavy Water on the Amino Acid Incorporation of Subcellular Liver Particles

I. Achátz: Freeze-Etching Investigation of the Ultrastructure of Striated Myofibrils (Preliminary Report)

K. SzÁsz, I. HoRváth: The in vivo Absorption of Chlorophyll-b in Higher Plants (Short Communication)

BooK REVIEW

ACTA BIOCHIMICA ET BIOPHYSICA erscheint vierteljährlich in Heften zu einem Band von etwa 400—500 Seiten. Format: $17 \times 25 \mathrm{~cm}$.

Abonnementspreis pro Band: $\$ 16.00 ; \mathrm{DM} 64,-$

Vertrieb: KULTURA, Budapest 62, Postfach 142; Auslieferung für das Gebiet der Deutschen Bundesrepublik: KUNST UND WISSEN, Erich Bieber, Stuttgart S., Wilhelmstraße 4

AKADÉMIAI KIADÓ, Verlag der Ungarischen Akademie der Wissenschaften, Budapest 502. Postfach 24 